\title{
Statistical Properties of the Transverse-Motion Energy Levels for Channeling Electrons in a Silicon Crystal under Dynamical Chaos Conditions
}

\author{
V. V. Syshchenko ${ }^{a, *}$ and A. I. Tarnovsky ${ }^{a}$ \\ ${ }^{a}$ Belgorod National Research University, Belgorod, 308015 Russia \\ *e-mail: syshch@yandex.ru \\ Received December 30, 2020; revised January 28, 2021; accepted January 30, 2021
}

\begin{abstract}
The classical motion of particles in external fields is frequently chaotic although deterministic within the classical limit. Studying the quantum mechanical behavior of such systems amounts to the problematics of quantum chaos. Quantum chaos manifests itself primarily in the statistical properties of the energy-level arrays of such systems. However, the typical objects under consideration are systems of the type billiards with rigid walls. This paper studies the calculated energy levels of the transverse motion of relativistic electrons in the axial-channeling regime along the [100] direction of the silicon crystal which is described as motion in the smooth potential well. The nearest-level spacing distributions as well as the spectral rigidity are studied for the range of parameters where electron motion is chaotic within the classical limit. Both these characteristics demonstrate agreement with quantum-chaos-theory predictions.
\end{abstract}

Keywords: chaotic dynamics, quantum chaos, channeling, energy levels, nearest-neighbor spacing distribution, Wigner distribution, spectral rigidity

DOI: $10.1134 /$ S 1027451021040200

\section{INTRODUCTION}

The essence of the problem of quantum chaos lies in the search for distinctive features of the behavior of quantum systems with chaotic dynamics in the classical limit [1-4]. One of these features is the statistical properties of the energy levels of a quantum system. Systems with regular dynamics lack correlations between different energy levels, which leads to an exponential distribution of the distances $s$ between adjacent levels with a maximum at $s=0$, which is characteristic of the Poisson flow. In chaotic systems, correlations between different levels lead to their mutual repulsion; in this case, the interlevel distances obey the Wigner distribution:

$$
p(s)=\left(\pi \rho^{2} s / 2\right) \exp \left(-\pi \rho^{2} s^{2} / 4\right),
$$

where $\rho$ is the average density of levels in the considered section of the energy spectrum of the system (which is assumed to be constant throughout the entire section). The presence of correlations between energy levels is also checked using $\Delta_{3}$ statistics [2-5].

In [6], the predictions of the theory of quantum chaos were tested for the energy levels of the transverse motion of relativistic electrons moving in the axialchanneling regime $[7,8]$ near the [110] direction of a silicon crystal. In this case, the statistics of energy levels was studied in a narrow interval near the upper edge of the potential well formed by continuous potentials of atomic chains, within which the average density of levels can be considered constant.

In this work, all energy levels of the transverse motion of electrons with an energy of longitudinal motion of $500 \mathrm{MeV}$ in such a well are found and their statistical properties are investigated over the entire range of transverse energies, within which classical dynamics is chaotic. In this case, the initial set of levels is subjected to the unfolding procedure [2, 4], as a result of which a new array of levels with a constant average density over the entire interval is obtained.

\section{EXPERIMENTAL}

When a relativistic charged particle is incident at a small angle to the crystallographic axis densely packed with atoms, the phenomenon of axial channeling is possible, when the particle performs finite motion in a plane perpendicular to the chain axis $[7,8]$. In this case, the discrete potential of the chain can be replaced with a continuous potential averaged along its length. In such a potential, the particle momentum $p_{\|}$ component parallel to the chain axis is retained, which makes it possible to describe the motion of a particle in the transverse plane using the two-dimensional Schrödinger equation

$$
\hat{H} \Psi(x, y)=E_{\perp} \Psi(x, y)
$$




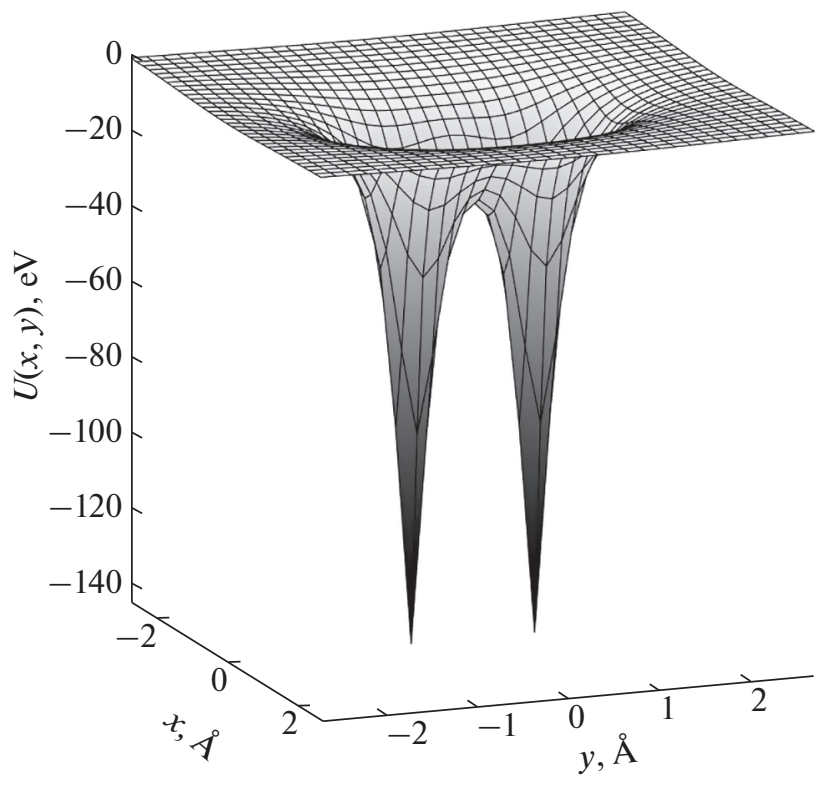

Fig. 1. Potential energy (5) of an electron in the field of two nearest chains [110] of a silicon crystal.

with the Hamiltonian operator

$$
\hat{H}=-\frac{\hbar^{2}}{2 E_{\|} / c^{2}}\left(\frac{\partial^{2}}{\partial x^{2}}+\frac{\partial^{2}}{\partial y^{2}}\right)+U(x, y),
$$

in which the role of the particle mass is played by the quantity $E_{\|} / c^{2}$, Where $E_{\|}=\left(m^{2} c^{4}+p_{\|} c^{2}\right)^{1 / 2}$ is the energy of longitudinal motion [7].

The continuous potential of an individual atomic chain can be approximated by the formula [7]:

$$
U_{1}(x, y)=-U_{0} \ln \left(1+\frac{\beta R^{2}}{x^{2}+y^{2}+\alpha R^{2}}\right),
$$

where for the [110] chain of a silicon crystal $U_{0}=60 \mathrm{eV}$, $\alpha=0.37, \beta=3.5$, and $R=0.194 \AA$ (Thomas-Fermi radius). The distance between the two nearest [110] chains in a silicon crystal is $a / 4$, where $a=5.431 \AA$ is the lattice period of the crystal. Taking this into account and neglecting the influence of distant chains, the continuous potential will have the form

$$
U(x, y)=U_{1}(x, y+a / 8)+U_{1}(x, y-a / 8) .
$$

The symmetry of potential (5) with respect to planes $x=0$ and $y=0$ (Fig. 1) leads to the presence of four symmetry classes of wave functions, describing the eigenstate of an electron:

$$
\begin{gathered}
\left\{\begin{array}{l}
\Psi_{++}(-x, y)=\Psi_{++}(x, y) \\
\Psi_{++}(x,-y)=\Psi_{++}(x, y),
\end{array}\right. \\
\left\{\begin{array}{l}
\Psi_{+-}(-x, y)=\Psi_{+-}(x, y) \\
\Psi_{+-}(x,-y)=-\Psi_{+-}(x, y),
\end{array}\right.
\end{gathered}
$$

$$
\begin{aligned}
& \left\{\begin{array}{l}
\Psi_{-+}(-x, y)=-\Psi_{-+}(x, y) \\
\Psi_{-+}(x,-y)=\Psi_{-+}(x, y),
\end{array}\right. \\
& \left\{\begin{array}{l}
\Psi_{--}(-x, y)=-\Psi_{--}(x, y) \\
\Psi_{--}(x,-y)=-\Psi_{--}(x, y) .
\end{array}\right.
\end{aligned}
$$

Since the symmetry group is Abelian, all four types of eigenstates are nondegenerate [9]. Moreover, the statistical properties of energy levels for each of these classes must be investigated independently [3]. The energy levels of the transverse motion of a charged particle $E_{\perp}$ in the field (5) are found numerically using the spectral method [10], which was successfully used in the channeling problem in [6,11-14].

The statistical properties of arrays of levels of quantum systems, leading to distribution (1), describe fluctuations in the positions of levels relative to their average density, which, as a rule, is not constant [9]. To isolate these fluctuations from the actual location of the levels, a procedure called unfolding $[2,4]$ is used, which is as follows.

At the first step of the procedure, an integrated level density is introduced:

$$
N(E)=\int_{E_{\min }}^{E} \rho\left(E^{\prime}\right) d E^{\prime},
$$

where $\rho(E)=\sum_{\mathrm{i}} \delta\left(E-E_{\mathrm{i}}\right)$ is the actual level density $E_{\mathrm{i}}$ of the quantum system. The step function (10) can be represented as the sum of the smooth and fluctuating parts:

$$
N(E)=N_{\mathrm{av}}(E)+N_{\mathrm{fl}}(E) .
$$

In this work, the function $N_{\text {av }}(E)$ is approximated by a polynomial of the fifth degree. In the next step, it displays the initial energy levels:

$$
\widetilde{E_{\mathrm{i}}}=N_{\text {av }}\left(E_{\mathrm{i}}\right) .
$$

The resulting new sequence of levels $\widetilde{E}_{\mathrm{i}}$ will have a constant average density $\rho$ equal to one.

In addition to studying the distribution of distances between adjacent levels, the presence of correlations is also checked by calculating the so-called spectral rigidity, or $\Delta_{3}$ statistics [2-5]. For a set of unfolded levels in a certain interval

$$
\alpha \leq \widetilde{E}_{\mathrm{i}} \leq \alpha+L
$$

a step function is constructed $\tilde{N}(\tilde{E})$, similar to (10), and the variance of the deviation of this function from the straight line, fitted by the least-squares method, is calculated:

$$
\Delta_{3}(\alpha, L)=\frac{1}{L} \min _{A, B} \int_{\alpha}^{\alpha+L}(\tilde{N}(\tilde{E})-A \tilde{E}-B)^{2} d \tilde{E} .
$$

The average value of this value $\Delta_{3}(L)=\left\langle\Delta_{3}(\alpha, L)\right\rangle$ over a set of consecutive nonoverlapping intervals serves as 

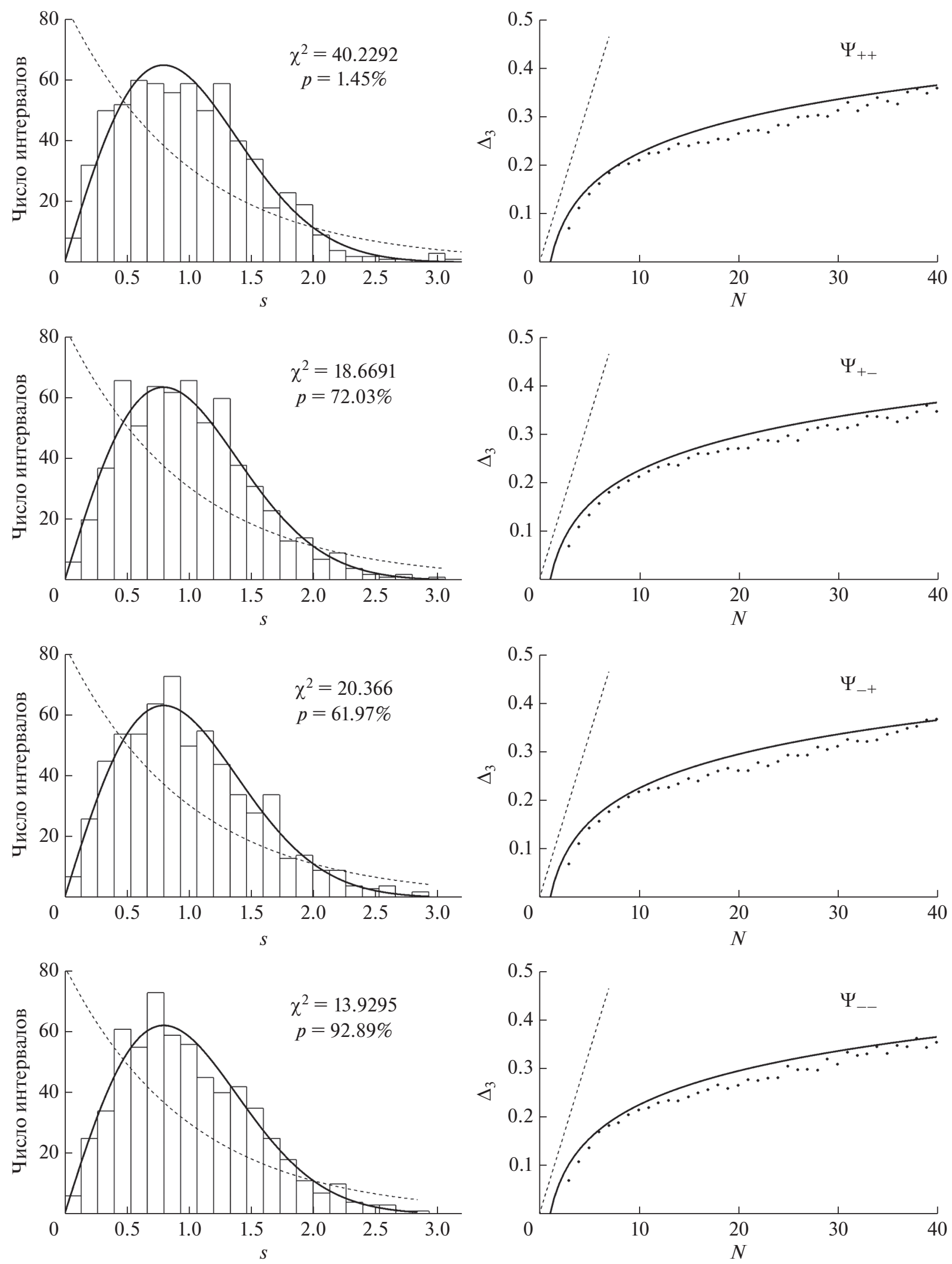

Fig. 2. Redistribution of distances between adjacent energy levels after the deployment procedure for the interval (16) of values of the energy of the transverse motion of an electron (histogram) for four classes of symmetry of the wave function (6) -(9); solid curves correspond to the Wigner distribution (1) - on the left. Results of calculating the spectral rigidity for the same arrays of unfolded levels; solid curves correspond to prediction (15). The dashed lines indicate the predictions of the theory for regular systems-on the right. 
a measure of the level correlation in the spectrum. For completely uncorrelated levels (Poisson flow characteristic of systems that are regular in the classical limit), the dependence of the quantity $\Delta_{3}$ on the number of levels $N$ in the range of width $L$ is described by the formula $\Delta_{3}(N)=N / 15$. For a chaotic quantum system, the spectral properties of which are described, as in our case, by a Gaussian orthogonal ensemble of random matrices [2-4], this quantity (also called the spectral rigidity) is described by the formula [4]:

$$
\Delta_{3}(N)=\frac{1}{\pi^{2}}\left(\ln (2 \pi N)+\gamma-\frac{5}{4}-\frac{\pi^{2}}{8}\right),
$$

where $\gamma=0.577216 \ldots$ is Euler's constant.

\section{RESULTS AND DISCUSSION}

The total number of energy levels of transverse motion in a potential well formed by continuous potentials of chains increases with an increase in the energy of longitudinal motion $E_{\|}$[7]; while choosing $E_{\|}=500 \mathrm{MeV}$ provides a sufficient number of levels to study their statistical properties.

The saddle point of the potential (5) is at a depth $29.38 \mathrm{eV}$. As the construction of Poincaré sections shows [7, 8], for the interval

$$
-29.38 \leq E_{\perp} \leq-3 \mathrm{eV}
$$

(from the saddle point to the upper edge of the potential well) the classical dynamics of an electron is chaotic for the overwhelming majority of the initial conditions (regular trajectories occupy less than $1 \%$ of the region of the phase space accessible for motion). Thus, it can be expected that the distribution of distances between neighboring energy levels after the deployment procedure will be described by the Wigner formula (1).

Figure 2 on the left shows the histograms of the distribution of interlevel distances on the interval (13) for states belonging to each of the four symmetry classes (6) - (9), as well as the curves corresponding to prediction (1). We see good agreement of the obtained distributions with the predictions of the theory of quantum chaos, which is also confirmed by the criterion $\chi^{2}$ (values $\chi^{2}$ and their corresponding $p$ values for 23 degrees of freedom are shown in the figures). On the right are the results of calculating the spectral rigidity, which also agree well with the predictions of the theory for systems exhibiting chaotic behavior in the classical limit.

\section{CONCLUSIONS}

All energy levels of the transverse motion of an electron with an energy of $500 \mathrm{MeV}$ moving in the mode of axial channeling in the [110] direction of $\phi$ silicon crystal were found numerically in this work. In this direction, pairs of closest parallel atomic chains create double-well potentials, above the saddle points of which the classical motion of an electron is chaotic for the overwhelming majority of initial conditions. For energy levels lying above the saddle point, two statistical properties are investigated: the distribution of the distances between the nearest neighboring levels and the spectral rigidity ( $\Delta_{3}$ Dyson-Mehta statistics). Both of these characteristics show good agreement with the predictions of quantum chaos theory for systems whose dynamics are completely chaotic in the classical limit.

\section{REFERENCES}

1. M. V. Berry, Proc. R. Soc. A 413, 183 (1987). https://doi.org/10.1098/rspa.1987.0109

2. O. Bohigas and M.-J. Giannoni, "Chaotic motion and random matrix theories," in Mathematical and Computational Methods in Nuclear Physics, Ed. by J. S. Dehesa, J. M. G. Gomez, and A. Polls (Springer, Berlin, 1984), Vol. 209, p. 1.

3. H.-J. Stöckmann, Quantum Chaos (Cambridge Univ. Press, Cambridge, 2000; Fizmatlit, Moscow, 2004).

4. L. E. Reichl, The Transition to Chaos. Conservative Classical Systems and Quantum Manifestations (Springer, New York, 2004; RKhD, Moscow, 2008).

5. F. J. Dyson and M. L. Mehta, J. Math. Phys. 4, 713 (1963). https://doi.org/10.1063/1.1704009

6. N. F. Shul'ga, V. V. Syshchenko, A. I. Tarnovsky, and A. Yu. Isupov, J. Surf. Invest.: X-Ray, Synchrotron Neutron Tech. 9, 721 (2015).

https://doi.org/10.1134/S1027451015040199

7. A. I. Akhiezer and N. F. Shul'ga, High-Energy Electrodynamics in Matter (Nauka, Moscow, 1993) [in Russian].

8. A. I. Akhiezer, N. F. Shul'ga, V. I. Truten', A. A. Grinenko, and V. V. Syshchenko, Phys.-Usp. 38, 1119 (1995). https://doi.org/10.1070/PU1995v038n10ABEH000114

9. L. D. Landau and E. M. Lifshits, Theoretical Physics, Vol. 3: Quantum Mechanics. Nonrelativistic Theory (Fizmatlit, Moscow, 2016) [in Russian].

10. M. D. Feit, J. A. Fleck, Jr., and A. Steiger, J. Comput. Phys. 47, 412 (1982). https://doi.org/10.1016/0021-9991(82)90091-2

11. N. F. Shul'ga, V. V. Syshchenko, and V. S. Neryabova, J. Surf. Invest.: X-Ray, Synchrotron Neutron Tech. 7, 279 (2013). https://doi.org/10.1134/S1027451013020183

12. N. F. Shul'ga, V. V. Syshchenko, and V. S. Neryabova, Nucl. Instrum. Methods Phys. Res., Sect. B 309, 153 (2013). https://doi.org/10.1016/j.nimb.2013.01.022

13. N. F. Shul'ga, V. V. Syshchenko, and V. S. Neryabova, Vopr. At. Nauki Tekh., Ser.: Yad.-Fiz. Issled. 5, 111 (2014).

14. N. F. Shul'ga, V. V. Syshchenko, A. I. Tarnovsky, and A. Yu. Isupov, Nucl. Instrum. Methods Phys. Res., Sect. B 370, 1 (2016). https://doi.org/10.1016/j.nimb.2015.12.040 